\title{
Weak Deflection Angle and Greybody Bound of Magnetized Regular Black Hole
}

\author{
Wajiha Javed, ${ }^{1, *}$ Sibgha Riaz, ${ }^{1, \dagger}$ and Ali Övgün ${ }^{2, \ddagger}$ \\ ${ }^{1}$ Department of Mathematics, Division of Science and Technology, University of Education, Lahore-54590, Pakistan \\ ${ }^{2}$ Physics Department, Eastern Mediterranean University, \\ Famagusta, 99628 North Cyprus via Mersin 10, Turkey.
}

(Dated: February 16, 2022)

\begin{abstract}
In this paper, we examine the weak deflection angle and greybody factor of magnetized regular black hole. We implement the Gauss-Bonnet theorem on magnetized regular black hole to get the deflection angle. Moreover, we analyze the influence of plasma and non-plasma mediums on deflection angle of magnetized regular black hole. We observe the graphical behaviour of plasma and non-plasma mediums on deflection angle of magnetized regular black hole. Lastly, we study the rigorous bound phenomenon of the greybody factor of magnetized regular black hole. For this purpose we calculate greybody factor results and analyze the graphical behaviour of greybody bound for the different values of the parameters.
\end{abstract}

PACS numbers: 95.30.Sf, 98.62.Sb, 97.60.Lf

Keywords: General Relativity; Gravitational Lensing; Magnetized Black Holes; Gauss-Bonnet Theorem; Plasma Medium; Greybody Factor

\section{INTRODUCTION}

Black Holes (BHs), a great prediction of Einstein's theory of General Relativity (GR) and of different metric theories of gravity, and at the same time the understandable objects inside the universe and are of most important for each classical and quantum gravity [1]. A region of space having a gravitational field so severe that no matter or radiation can escape is called Black Hole (BH). Initially BHs known as "collapser", the term derived from the collapse of the start, and after Wheeler put forward the term BH in 1967 [3]. A BH is a place in a space where gravity is strongly pulled and even light does not comes out. Gravity is very strong because matter is pushed into a small space. No light, so you can't see the BH. Space telescopes with special tools can help you to find BHs. A special tools can help us to see how a star very close to a BH behaves differently than other stars.

In 1916, Einstein anticipated the existence of gravitational lensing (GL) and gravitational wave as a part of concept of GR [1]. The gravitational waves were detected by Laser Interferometer Gravitational-wave Observatory (LIGO) in 2015, which indicated that theoretical predictions are well expressed with experimental observations [4], After detection of gravitational waves by LIGO, there is renewed interest in the topic of GL [5]. The method in which gravity deflect the light is called GL. Gravitational lensing is a helpful method to understand the dark matter, galaxies and universe. Such a lot of work were accomplished on GL for BHs, wormholes and different objects after the 1st statement of Eiddington. The topic of GL takes a tremendous attraction. The GL has been categorized withinside the literature as strong GL, weak GL, micro GL [6, 7].

Weak Gravitational lensing (WGL) is an effective tool to measure the masses of different objects in the universe. Weak gravitational lensing investigate the cause of elevated enlargement of the universe and additionally distinguish among modified gravity and dark energy. The method which is used to observed the magnification and position of a BHs is known as strong GL. Similarly, micro GL is worked on the bases of strong GL in which the image separation is too small to be resolved. The GL affect was first proposed by Soldner in 1801 in the background of Newtonian theory [8]. Eddington then used data obtained during the 1919 by solar eclipse to measure value close to the GR [9]. Gravitational lensing were the processed in different space time in different ways [10]-[20]. Moreover, many studies relating GL to Gauss-Bonnet Theorem (GBT) over the year, after gracefully demonstrating that Gibbons and Werner can use GBT abruptly to calculating deflection angles for flat statistic $\mathrm{BH}$ [21], which is given as:

$$
\alpha=-\iint_{D_{\infty}} \mathcal{K} d r
$$

Where $\alpha$ represents the deflection angle, $\mathcal{K}$ represents the Gaussian optical curvature, $d r$ represents the optical surface and $D_{\infty}$ symbolizes the infinite domain of the space.

\footnotetext{
*Electronic address: wajiha.javed@ue.edu.pk

†Electronic address: sibghariaz993@gmail.com

‡Electronic address: ali.ovgun@emu.edu.tr
} 
Afterwards, Werner extended GTB method to stationary BH [22]. Next Ishihara et al [23], stated that it is possible to find a finite distance of deflection angle by using optical Fermat geometry. Crisnejo and Gallo investigated the deflection angle of light in plasma medium [24]. Since then, there is a constantly increasing interest to the WGL through the Gibbons and Werner and GBT methodology deprive a unique prospect for BHs and wormholes [54]-[65]. In addition, Hensh et al. computed the GL of Kehagias-Stefsos dense particles under the influence of plasma[66].

In 1974 Hawking predicted that BHs can emitted quantum radiation. This radiation is termed as Hawking radiation [67]. This fact was inferred after analysing quantum field theory in BH. From quantum field theory, it is conceivable to produced the particles and to inhale the particles. When pair production take place close to the horizon of the $\mathrm{BH}$, one of the particle present in the pair production falls and the other particles leaves the BH, outside observers will detect this particle as Hawking radiation. However, according to the GR, a BH bends spacetime around itself. This spacetime behaves like a the gravitational potential for the particles to move. Few of the particles are reflected by the $\mathrm{BH}$ and remanning particles are transmitted through the $\mathrm{BH}$. As a result, the Hawking radiation that the observer observes from outside the $\mathrm{BH}$ is distinct from the propagation through the gravitational potential. This distinction is known as the greybody factor. There are many studies investigating the computation of the greybody factor [68]-[78]. If the gravitational potential is high enough, then we use the WKB approximation method to derived the greybody factor [78]. Another attractive ways to calculate the bound of greybody factor are [79]-[87]. The basis purpose of this paper is to deeply analyze how a deflection angle effect on the plasma and non-plasma medium of a magnetized regular black hole $(\mathrm{MRBH})$. We find the deflection angle of plasma and non-plasma mediums and also observed its greybody bound and study its graphical behaviour.

This work is organized as, In section 2, we study MRBH. In section 3, by using GBT, we prove the deflection angle in the background of non-plasma medium. In section 4, we analyze the graphical behaviour of deflection angle in non-plasma medium. In section 5, we compute the deflection angle for MRBH in plasma medium, and in section $\mathbf{6}$ we study the graphical influence of deflection angle in plasma medium. In the section 7, we investigate and calculate the bound of greybody factor for MRBH. In the section $\mathbf{8}$ we observe greybody graphical impact. The last section $\mathbf{9}$ is denoted to express the conclusion.

\section{MAGNETIZED REGULAR BLACK HOLE (MRBH)}

The MRBH metric in spherically coordinates is given below [88],

$$
d s^{2}=-H(r) d t^{2}+\frac{d r^{2}}{H(r)}+r^{2} d \Omega_{2}^{2}
$$

$H(r)$ can be defined as,

$$
H(r)=1-\frac{2 G m}{r}+\frac{G q^{2}}{r^{2}}+\frac{4 G^{2} m^{2} l^{2}}{r^{4}}-\frac{4 G^{2} m l^{2} q^{2}}{r^{5}}+\mathcal{O}\left(r^{-6}\right) \quad r \rightarrow \infty,
$$

where

$$
d \Omega_{2}^{2}=d \theta^{2}+\sin ^{2} \theta d \phi^{2}
$$

Here $G$ is a Newton constant, $l$ is the fundamental length and $m$ is mass which is constant and $q$ is a magnetic charge. The optical metric is written in equatorial plane as $\left(\theta=\frac{\pi}{2}\right)$. To get null geodesic $d s^{2}=0$.

$$
d t^{2}=\frac{d r^{2}}{\left(1-\frac{2 G m}{r}+\frac{G q^{2}}{r^{2}}+\frac{4 G^{2} m^{2} l^{2}}{r^{4}}-\frac{4 G^{2} m l^{2} q^{2}}{r^{5}}\right)^{2}}+\frac{r^{2} d \phi^{2}}{1-\frac{2 G m}{r}+\frac{G q^{2}}{r^{2}}+\frac{4 G^{2} m^{2} l^{2}}{r^{4}}-\frac{4 G^{2} m l^{2} q^{2}}{r^{5}}} .
$$

The Gaussian curvature can be define by using below expression;

$$
\mathcal{K}=\frac{\text { RicciScalar }}{2} .
$$


The required Gaussian optical curvature of the optical metric for the MRBH is obtained as defined below;

$$
\begin{aligned}
\mathcal{K} & \approx \frac{\left(3 q^{2}-2 m r\right) G}{r^{4}}+\frac{\left(-150 m q^{2}+100 m^{2} r+2 q^{4} r-6 m q^{2} r^{2}+3 m^{2} r^{3}\right) G^{2}}{r^{7}} \\
& -\frac{2\left(47 m q^{4}+9 l^{2} m q^{4}-150 m^{2} q^{2} r-15 l^{2} m^{2} q^{2} r+78 m^{3} r^{2}+6 l^{2} m^{3} r^{2}\right) G^{3}}{r^{9}} \\
& -\frac{2\left(485 m^{2} q^{4}-360 l^{2} m^{2} q^{4}-780 m^{3} q^{2} r+600 l^{2} m^{3} q^{2} r+6 m q^{6} r\right) G^{4}}{r^{12}} \\
& -\frac{2\left(-3 l^{2} m q^{6} r+312 m^{4} r^{2}-240 l^{2} m^{4} r^{2}-18 m^{2} q^{4} r^{2}+9 l^{2} m^{2} q^{4} r^{2}\right) G^{4}}{r^{12}} \\
& -\frac{2\left(18 m^{3} q^{2} r^{3}-9 l^{2} m^{3} q^{2} r^{3}-6 m^{4} r^{4}+3 l^{2} m^{4} r^{4}\right) G^{4}}{r^{12}} .
\end{aligned}
$$

\section{WEAK DEFLECTION ANGLE OF MRBH FOR NON-PLASMA MEDIUM}

According to GBT the topology of domain $\left(\mathcal{S}_{R}\right)$ and the intrinsic structural of the spacetime are connected with boundary $\partial \mathcal{S}_{R}$. Then, with the help of GBT angle of deflection of the desired BH can be calculated,

$$
\iint_{\mathcal{S}_{R}} \mathcal{K} d S+\oint_{\partial \mathcal{S}_{R}} k d t+\sum_{i} \epsilon_{i}=2 \pi \mathcal{X}\left(\mathcal{S}_{R}\right)
$$

$\mathcal{K}$ denotes the optical Gaussian curvature and $k$, denotes the geodesic curvature revealed as $k=\bar{h}\left(\nabla_{\dot{\beta}} \dot{\beta}, \ddot{\beta}\right)$ such that $\bar{h}(\dot{\beta}, \dot{\beta})=$ 1. If $\beta$ is considered to be a smooth curve then we obtained $\dot{\beta}$ as a unit speed vector. $\epsilon_{i}$ is represented the exterior angle at the $i t h$ vertex. As $R \rightarrow \infty$, then we take $\pi / 2$ as jump angles. This gives us $\left(\theta_{\mathcal{S}}+\theta_{\mathcal{O}} \rightarrow \pi\right)$.

$$
\iint_{\mathcal{S}_{R}} \mathcal{K} d S+\oint_{\partial \mathcal{S}_{R}} k d t+\epsilon_{i}=2 \pi \mathcal{X}\left(\mathcal{S}_{R}\right),
$$

Here, the total jump angle is presented by $\epsilon_{i}=\pi$. $\mathcal{X}$ is representing Euler characteristic number which is 1 . Geodesic curvature takes the form $k\left(C_{R}\right)=\left|\nabla_{\dot{P}_{R}} \dot{P}_{R}\right|$ that we will compute. The radial part mention in the geodesic curvature can be calculated as:

$$
\left(\nabla_{\dot{P}_{R}} \dot{P}_{R}\right)^{r}=\dot{P}_{R}^{\phi} \partial_{\phi} \dot{P}_{R}^{r}+\beta_{\phi \phi}^{r}\left(\dot{P}_{R}^{\phi}\right)^{2}
$$

For high value of $R, P_{R}:=r(\phi)=R=$ const, where $R$ shows the distance from the coordinate origin. Last expression $\beta_{\phi \phi}^{r}$ shows the Christofell symbols in connection to the optical geometry. Due to the non-presence of the topological effect the first term present in the above expression vanishes while the second term takes the form $k\left(P_{R}\right)=\left|\nabla_{\dot{P}_{R}} \dot{P}_{R}\right|$ that shall be computed by using of unit speed term.

$$
\left(\nabla_{\dot{P}_{R}^{r}} \dot{P}_{R}^{r}\right)^{r} \rightarrow \frac{1}{R}
$$

$k\left(P_{R}\right) \rightarrow R^{-1}$ due to absences of topological effect. By taking the advantage of the optical metric; it can be written $d t=R d \phi$. It can be stated that:

$$
k\left(P_{R}\right) d t=\frac{1}{R} R d \phi
$$

The following expression can be written when we collect all the above results;

$$
\iint_{\mathcal{S}_{R}} \mathcal{K} d s+\oint_{\partial \mathcal{S}_{R}} k d t \stackrel{R \rightarrow \infty}{=} \iint_{O_{\infty}} \mathcal{K} d S+\int_{0}^{\phi+\gamma} d \phi .
$$

The photon ray is expressed as $r(t)=b / \sin \phi$ at $0 t h$ order in weak field deflection limit. Therefore the angle of deflection is defined as:

$$
\gamma=-\int_{0}^{\pi} \int_{b / \sin \phi}^{\infty} \mathcal{K} \sqrt{\operatorname{det} \bar{g}} d r d \phi
$$


$\sqrt{\operatorname{det} \bar{g}}$ can be stated as:

$$
\sqrt{\operatorname{det} \bar{g}}=r\left(1-\frac{2 G m}{r}+\frac{G q^{2}}{r^{2}}+\frac{4 G^{2} m^{2} l^{2}}{r^{4}}-\frac{4 G^{2} m l^{2} q^{2}}{r^{5}}\right) d r
$$

By exploit the above relation and putting the values of Gaussian curvature into Eq.(13), angle of deflection $\gamma$ can be written as;

$$
\begin{aligned}
\gamma & \approx \frac{(4 G m)}{b}+\frac{\left(3 G^{2} m^{2} \pi\right)}{4 b^{2}}-\frac{\left(3 G q^{2} \pi\right)}{4 b^{2}}-\frac{\left(8 G^{2} m q^{2}\right)}{3 b^{3}}-\frac{\left(75 G^{2} m^{2} \pi\right)}{8 b^{4}} \\
& -\frac{\left(45 G^{3} m^{2} q^{2} \pi\right)}{32 b^{4}}+\frac{\left(15 G^{2} q^{4} \pi\right)}{64 b^{4}}+\frac{\left(32 G^{2} m q^{2}\right)}{b^{5}}+\frac{\left(12 G^{3} m q^{4}\right)}{5 b^{5}} \\
& +\frac{\left(125 m^{2} G^{3} q^{2} \pi\right)}{8 b^{6}}+\frac{\left(525 G^{4} m^{2} q^{4} \pi\right)}{256 b^{6}}-\frac{\left(4192 G^{3} m q^{4}\right)}{245 b^{7}}-\frac{\left(43155 G^{4} m^{2} q^{4} \pi\right)}{2048 b^{8}} \\
& +\frac{\left(6111 G^{4} m^{2} q^{4} \pi\right)}{256 b^{10}}+\frac{\left(567 G^{4} l^{2} m^{2} q^{4} \pi\right)}{128 b^{10}}
\end{aligned}
$$

\section{GRAPHICAL REPRESENTATION FOR NON-PLASMA OF MRBH}

This portion is for the graphical impact of the deflection angle $\gamma$ on MRBH. The physical importance of the graphs is too studied in order to analyze the effect of different parameters on the achieved angle by allocating different values to BH charge, impact parameters and fundamental length.

(i)

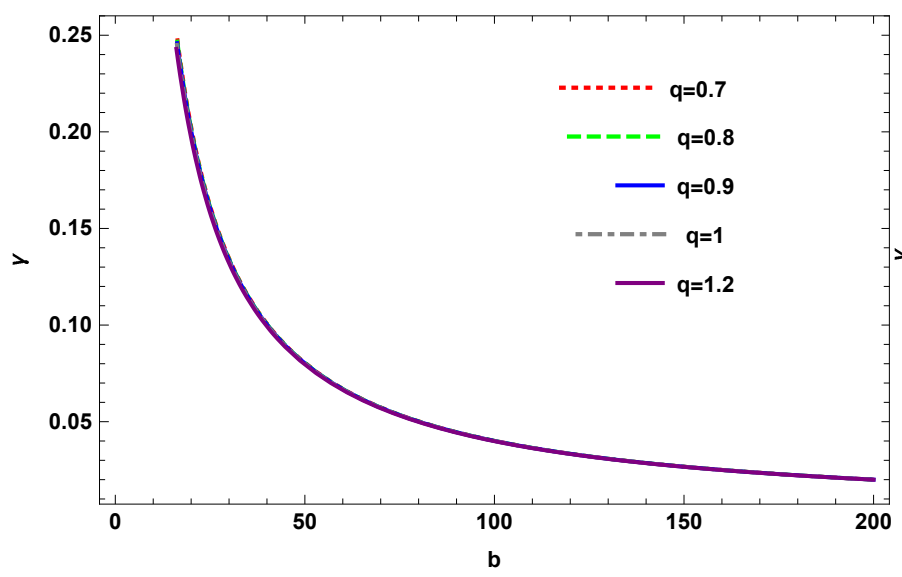

(i)

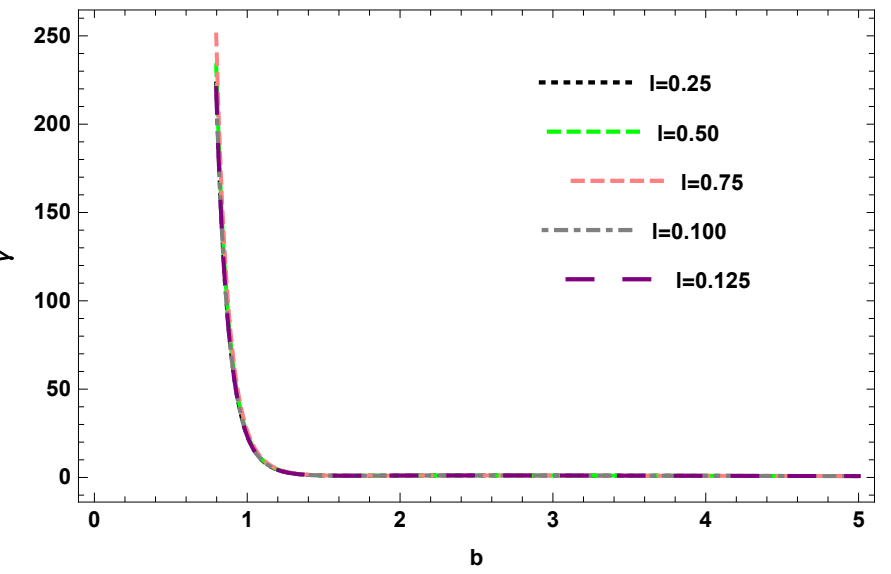

Figure 1: $\gamma$ in connection with $b$.

(i)

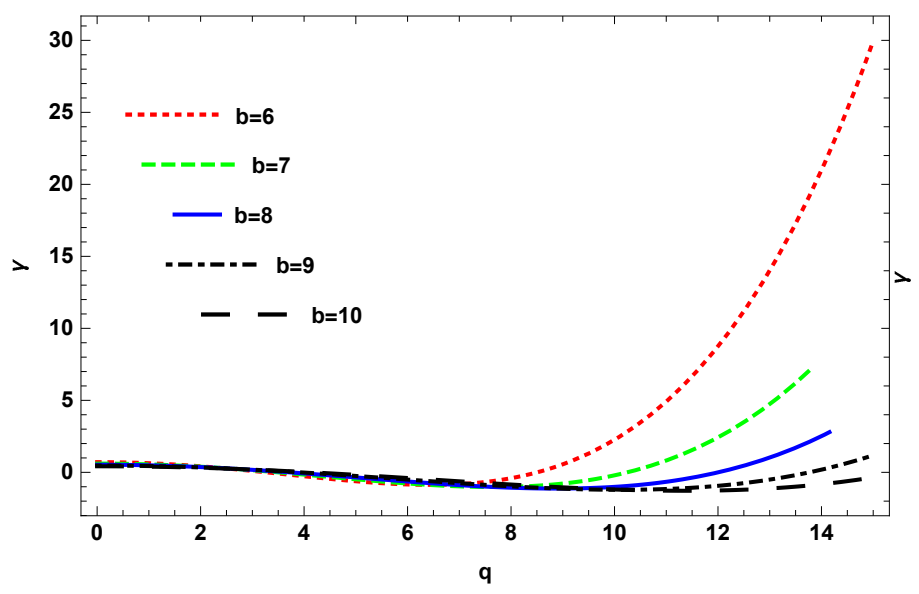

(i)

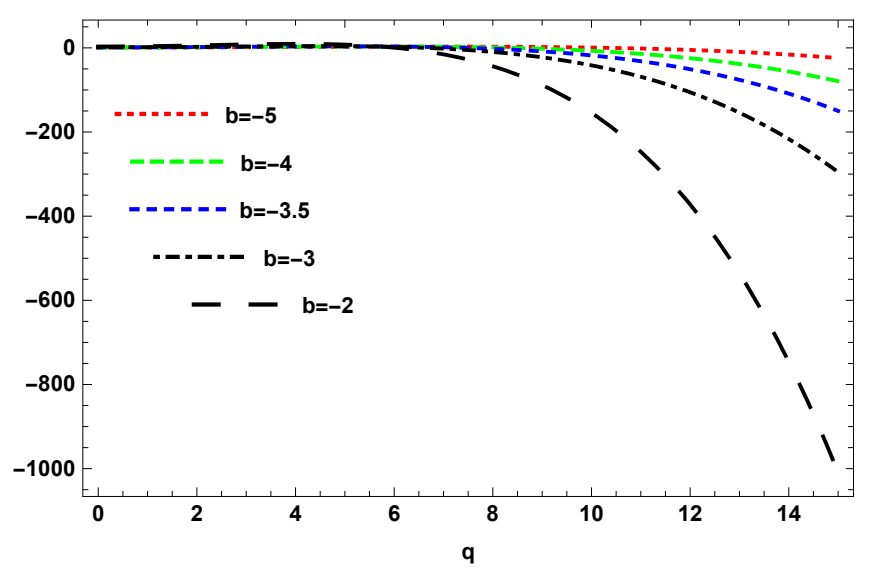

Figure 2: $\gamma$ in connection with $q$. 


\section{A. Angle of Deflection $\gamma$ in Connection with Impact Parameter $b$}

- Fig 1 shows the conduct of $\gamma$ with $b$ by taking the fix value $m, G$ and varying $q$ and $l$ respectively.

1. In the first figure, it is observed that obtained angle is constantly uniform when we made small changing of $q$. Impact parameter domain is taken to be $0 \leq b \leq 200$

2. In the second figure, it is observed that obtained angle in case of variations of $l$ is also uniform constant. Impact parameter domain is taken to be $0 \leq b \leq 5$.

3. In both figures angle shoes the same behaviour.

\section{B. Deflection Angle $\gamma$ in Connection with BH Charge $q$}

- Fig 2 shows the behaviour of $\gamma$ with $q$ by fixing $l, G, m$ and varying $b$. The domain of BH charge is taken to be $0 \leq b \leq 15$.

1. In the left graph, it can be observed that deflection angle is rapidly decreasing by making huge changing to $b$. $\gamma$ is quickly reduce at changing $6<b<7$.

2. In the right graph, we examined that attained angle $\gamma$ is negatively decreases when we change the negative values of b.

\section{EFFECT OF PLASMA ON WEAK DEFLECTION ANGLE $\gamma$ OF MRBH}

In this portion the impact of the angle of deflection by MRBH in plasma medium is examine. We consider the MRBH in plasma possessing refractive index,

$$
n^{2}(r)=1-\frac{\omega_{e}^{2}(r)}{\omega_{\infty}^{2}(r)}
$$

The corresponding refractive index can be stated as:

$$
n(r)=\sqrt{1-\frac{\omega_{e}^{2}}{\omega_{\infty}^{2}} H(r)}
$$

Here, $\omega_{e}$ denotes the electron plasma frequency and $\omega_{\infty}$ denotes photon frequency. The corresponding metric function is written as:

$$
d s^{2}=-H(r) d t^{2}+\frac{1}{H(r)} d r^{2}+r^{2} d \Omega_{2}^{2},
$$

and

$$
H(r)=1-\frac{2 G m}{r}+\frac{G q^{2}}{r^{2}}+\frac{4 G^{2} m^{2} l^{2}}{r^{4}}-\frac{4 G^{2} m l^{2} q^{2}}{r^{5}} .
$$

Since the source and observer are in the tropical region possess $\left(\theta=\frac{\pi}{2}\right)$. We imposed $d s^{2}=0$ in case of null geodesics.

$$
d \sigma^{2}=g_{x y}^{o p t} d x^{x} d x^{y}=n^{2}\left[\frac{d r^{2}}{H^{2}(r)}+\frac{r^{2} d \vartheta^{2}}{H(r)}\right]
$$

Gaussian optical curvature $\mathcal{K}$ can be expressed as;

$$
\mathcal{K}=\frac{R_{r \phi r \phi}\left(g_{x y}^{o p t}\right)}{\operatorname{det}\left(g_{x y}^{o p t}\right)}
$$

we let

$$
\omega=\frac{\omega_{e}}{\omega_{\infty}}
$$


By using Eq.(21) in the weak field approximation Gaussian curvature can be expressed as:

$$
\begin{aligned}
\mathcal{K} & \approx\left(-\frac{2}{r^{3}}-\frac{3 \omega^{2}}{r^{3}}\right) m G+\left(\frac{3}{r^{4}}+\frac{5 \omega^{2}}{r^{4}}\right) q^{2} G+\left(\frac{100+3 r^{2}}{r^{6}}+\frac{12\left(15+r^{2}\right) \omega^{2}}{r^{6}}\right) m^{2} G^{2} \\
& +\left(\frac{-6\left(25+r^{2}\right)}{r^{7}}-\frac{\left(275+26 r^{2}\right) \omega^{2}}{r^{7}}\right) m q^{2} G^{2}+\left(\frac{2}{r^{6}}+\frac{(10) \omega^{2}}{r^{6}}\right) q^{4} G^{2}-\frac{12\left(13+l^{2}\right)}{r^{7}} m^{3} G^{3} \\
& -\frac{2\left(362+13 l^{2}+6 r^{2}\right) \omega^{2}}{r^{7}} m^{3} G^{3}+\frac{30\left(10+l^{2}\right)}{r^{8}} m^{2} q^{2} G^{3}+\frac{2\left(737+34 l^{2}+16 r^{2}\right) \omega^{2}}{r^{8}} m^{2} q^{2} G^{3} \\
& \left.-\frac{2\left(47+9 l^{2}\right)}{r^{9}} m q^{4} G^{3}-\frac{\left(560+42 l^{2}+23 r^{2}\right) \omega^{2}}{r^{9}}\right) m q^{4} G^{3} \\
& +\frac{6\left(260-200 l^{2}-6 r^{2}+3 l^{2} r^{2}\right)}{r^{11}} m^{3} q^{2} G^{4}-\frac{2\left(1212+1592 l^{2}+941 r^{2}+75 l^{2} r^{2}\right) \omega^{2}}{r^{11}} m^{3} q^{2} G^{4} \\
& -\frac{2\left(485-360 l^{2}-18 r^{2}+9 l^{2} r^{2}\right)}{r^{12}} m^{2} q^{4} G^{4}+\frac{4\left(370+480 l^{2}+336 r^{2}+35 l^{2} r^{2}\right) \omega^{2}}{r^{12}} m^{2} q^{4} G^{4}
\end{aligned}
$$

By using GBT deflection angle is calculated and contrast to the equation of angle obtained for non-plasma. Since light beams follows a straight line approximation and calculate the deflection angle for plasma.

$$
\gamma=-\lim _{R \rightarrow 0} \int_{0}^{\pi} \int_{\frac{b}{\sin \phi}}^{R} \mathcal{K} d S .
$$

By using Eq.(24), we obtained the angle of the desired $\mathrm{BH}$ for plasma medium expresses as;

$$
\begin{aligned}
\gamma & \approx \frac{(4 G m)}{b}+\frac{\left(3 G^{2} m^{2} \pi\right)}{4 b^{2}}-\frac{\left(3 G q^{2} \pi\right)}{4 b^{2}}-\frac{\left(8 G^{2} m q^{2}\right)}{3 b^{3}}-\frac{\left(75 G^{2} m^{2} \pi\right)}{8 b^{4}}-\frac{\left(45 G^{3} m^{2} q^{2} \pi\right)}{32 b^{4}} \\
& +\frac{\left(15 G^{2} q^{4} \pi\right)}{64 b^{4}}+\frac{\left(32 G^{2} m q^{2}\right)}{b^{5}}+\frac{\left(12 G^{3} m q^{4}\right)}{5 b^{5}}+\frac{\left(125 m^{2} G^{3} q^{2} \pi\right)}{8 b^{6}}+\frac{\left(525 G^{4} m^{2} q^{4} \pi\right)}{256 b^{6}} \\
& -\frac{\left(4192 G^{3} m q^{4}\right)}{245 b^{7}}-\frac{\left(43155 G^{4} m^{2} q^{4} \pi\right)}{2048 b^{8}}+\frac{\left(6111 G^{4} m^{2} q^{4} \pi\right)}{256 b^{10}}+\frac{\left(567 G^{4} l^{2} m^{2} q^{4} \pi\right)}{128 b^{10}} \\
& +\frac{(2 G m) \omega^{2}}{b}-\frac{\left(15 G^{2} m^{2} \pi\right) \omega^{2}}{2 b^{4}}-\frac{\left(G^{2} m^{2} \pi\right) \omega^{2}}{2 b^{2}}+\frac{\left(80 G^{2} m q^{2}\right) \omega^{2}}{3 b^{5}}+\frac{\left(2 G^{2} m q^{2}\right) \omega^{2}}{b^{3}} \\
& -\frac{\left(G q^{2} \pi\right) \omega^{2}}{2 b^{2}}-\frac{\left(465 G^{3} m^{2} q^{2} \pi\right) \omega^{2}}{32 b^{6}}+\frac{\left(3 G^{3} m^{2} q^{2} \pi\right) \omega^{2}}{8 b^{4}}+\frac{\left(4112 G^{3} m q^{4}\right) \omega^{2}}{245 b^{7}}-\frac{\left(2 G^{3} m q^{4}\right) \omega^{2}}{3 b^{5}} \\
& -\frac{\left(3 G^{2} q^{4} \pi\right) \omega^{2}}{16 b^{4}}-\frac{\left(15435 G^{4} m^{2} q^{4} \pi\right) \omega^{2}}{256 b^{10}}+\frac{\left(14105 G^{4} m^{2} q^{4} \pi\right) \omega^{2}}{2048 b^{8}}-\frac{\left(45 G^{4} m^{2} q^{4} \pi\right) \omega^{2}}{128 b^{6}} \\
& +\frac{\left(945 G^{4} l^{2} m^{2} q^{4} \pi\right) \omega^{2}}{256 b^{10}} .
\end{aligned}
$$

It is clear from the above expression that if we consider $\omega=0$ then it decreases to the non-plasma equation and the influence of plasma is eliminated.

\section{GRAPHICAL ANALYSIS FOR PLASMA MEDIUM}

This portion is to study the effect of plasma on deflection angle $\gamma$. The physical importance of these figure are studied. Moreover, we consider $\omega=10^{-1}$ and give different values to the impact parameter $b$, charge $q$ of the $\mathrm{BH}$, to investigate the behaviour of $\gamma$. 
(ii)

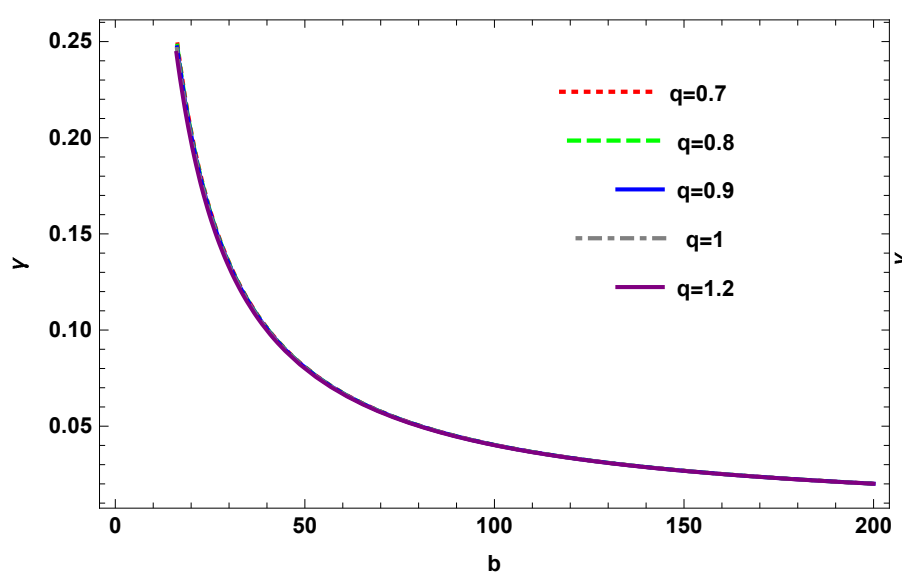

(ii)

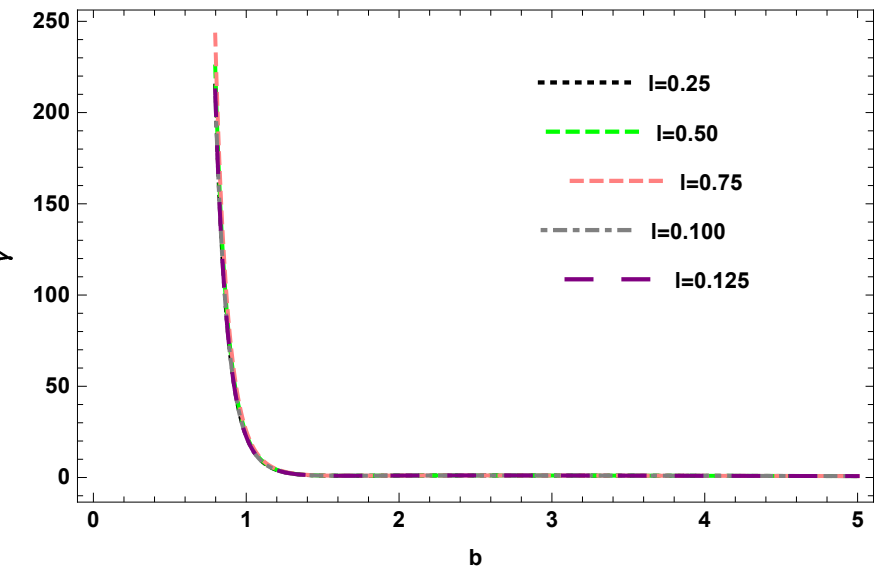

Figure 1: $\gamma$ in connection with $b$.

(ii)

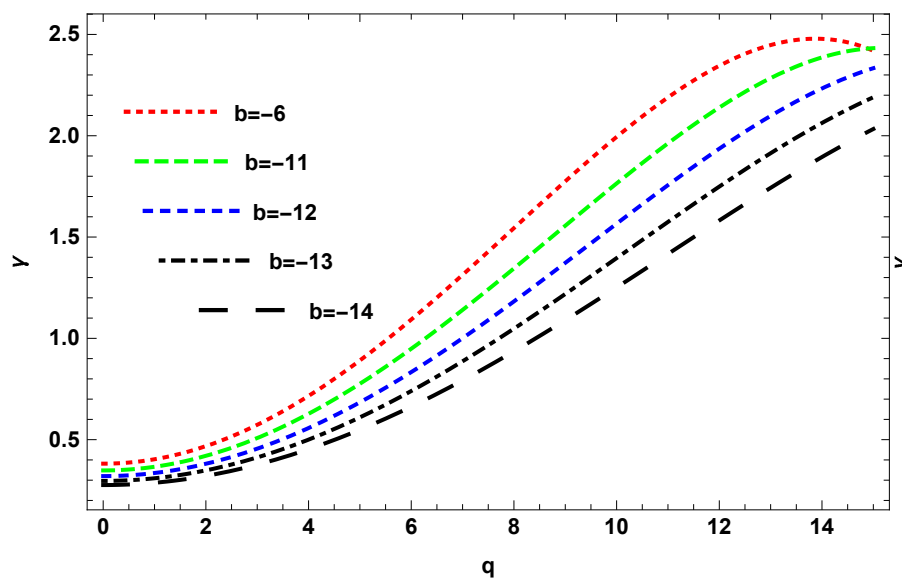

(ii)

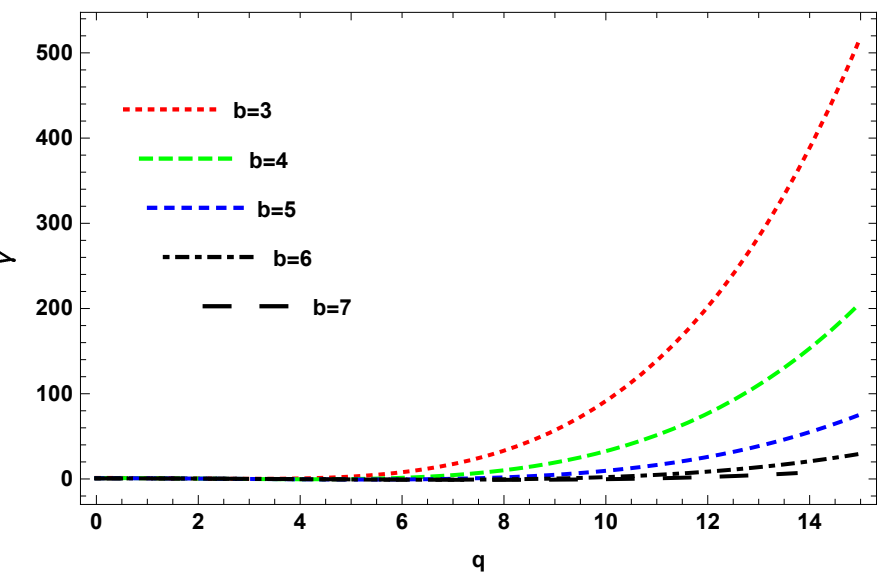

Figure 2: $\gamma$ in connection with $q$.

A. Deflection Angle $\gamma$ with Impact Parameter $b$

- Fig 1 shows the behavior of $\gamma$ with $b$ by giving a single value to $G$ and $m$ and changing the value of $q$ and $l$.

1. The first figure shows $\gamma$ with $b$. The angle of deflection is uniformly constant for small values of $q$.

2. The second figure shows that the attained angle $\gamma$ for fixed $q$ and small variations of $l$ is constantly uniform.

\section{B. Deflection Angle $\gamma$ with BH Charge $q$}

- Fig 2 exhibits the conduct of $\gamma$ with $q$ by assigning a single value to $l, G, m$ and changing $b$. It exhibits the behavior of $\gamma$ by assigning a fix value to $G, l, m$ and variant $b$ respectively .

1. We see that in the first figure the obtained angle has small variations of $b$ is smoothly decreasing.

2. We see that in the second figure the attained angle for small variations of $b$ is gradually decreasing.

\section{DERIVATION OF GREYBODY FACTOR OF MRBH}

The MRBH metric in D-dimension is defined as,

$$
d s^{2}=-H(r) d t^{2}+\frac{d r^{2}}{H(r)}+r^{2}\left(d \theta^{2}+\sin ^{2} \theta d \phi^{2}\right),
$$


Here the metric function is stated as:

$$
H(r)=1-\frac{2 G m}{r}+\frac{G q^{2}}{r^{2}}+\frac{4 G^{2} m^{2} l^{2}}{r^{4}}-\frac{4 G^{2} m l^{2} q^{2}}{r^{5}} .
$$

We computed numerically the horizons of the metric $H(r)$.

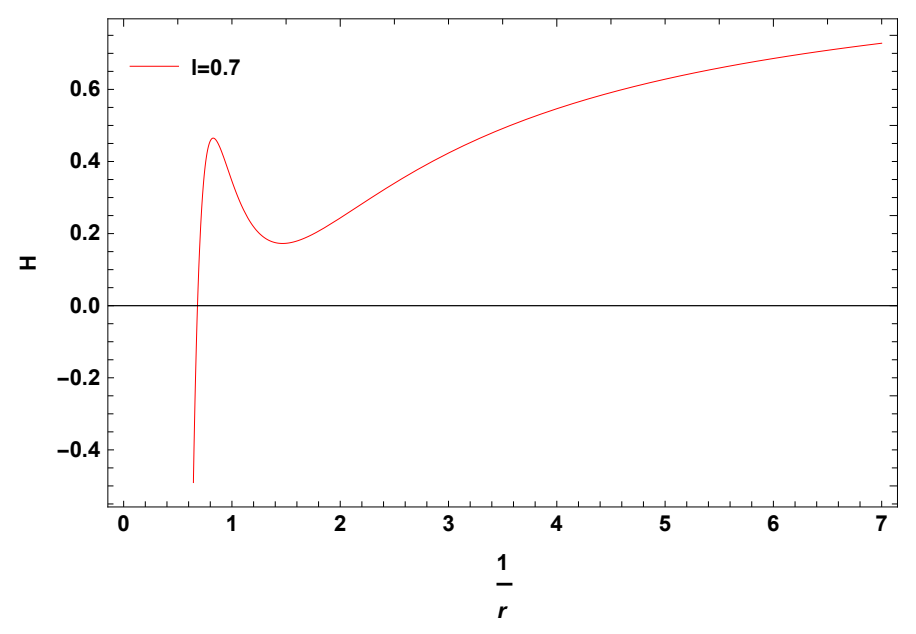

Figure a: $H$ vs $r$.

After the computation, the outer horizon of MRBH is $r_{+}=1.6$. Schrodinger like equation are defined as:

$$
\left[\frac{d^{2}}{d r_{*}^{2}}+\omega^{2}-\mathrm{V}(r)\right] \phi=0
$$

where

$$
d r_{*}=\frac{1}{H(r)} d r
$$

$\mathrm{V}(r)$ mention in the above equation is stated as:

$$
V(r)=\frac{(d-2)(d-4)}{4} \frac{H^{2}(r)}{r^{2}}+\frac{(d-2)}{2} \frac{H(r) \partial_{r} H(r)}{r}+l(l+d-3) \frac{H(r)}{r^{2}} .
$$

Greybody factor bound is defined as,

$$
T \geq \operatorname{sech}^{2}\left(\frac{l}{2 \omega} \int_{r_{+}}^{\infty} \frac{V(r)}{H(r)} d r_{*}\right)
$$

Then

$$
T \geq \operatorname{sech}^{2}\left(\frac{1}{2 \omega} \int_{r_{+}}^{\infty}\left(\frac{(d-2)(d-4)}{4} \frac{H(r)}{r^{2}}+\frac{(d-2)}{2} \frac{\partial_{r} H(r)}{r}+\frac{l(l+d-3)}{r^{2}}\right) d r\right)
$$

The above equation is written as when we put $\mathrm{d}=4$,

$$
T \geq \operatorname{sech}^{2}\left(\frac{1}{2 \omega} \int_{r_{+}}^{\infty}\left(\frac{\partial_{r} H(r)}{r}+\frac{l(l+1)}{r^{2}}\right) d r\right)
$$

After simplifying the integral, we substituted $r_{+}$,

$$
T \geq \operatorname{sech}\left[\frac{0.625 l(l+1)+0.390625 G m-0.305176 G^{2} l^{2} m^{2}-0.16276 G q^{2}+0.198682 G^{2} l^{2} m q^{2}}{2 \omega}\right]^{2}
$$

Hence we obtained the final expression for rigorous bound of the MRBH. 


\section{GRAPHICAL STUDY OF THE GREYBODY BOUND OF MRBH}

In this section we analyze the graphical behaviour of greybody bound on MRBH. The physical significance of these plots is also shows the effect of parameters on the lower bound by changing the values of the charge of the $\mathrm{BH}$.

\section{A. Rigorous bound $T_{b}$ in connection with omega $\omega$}

(i)

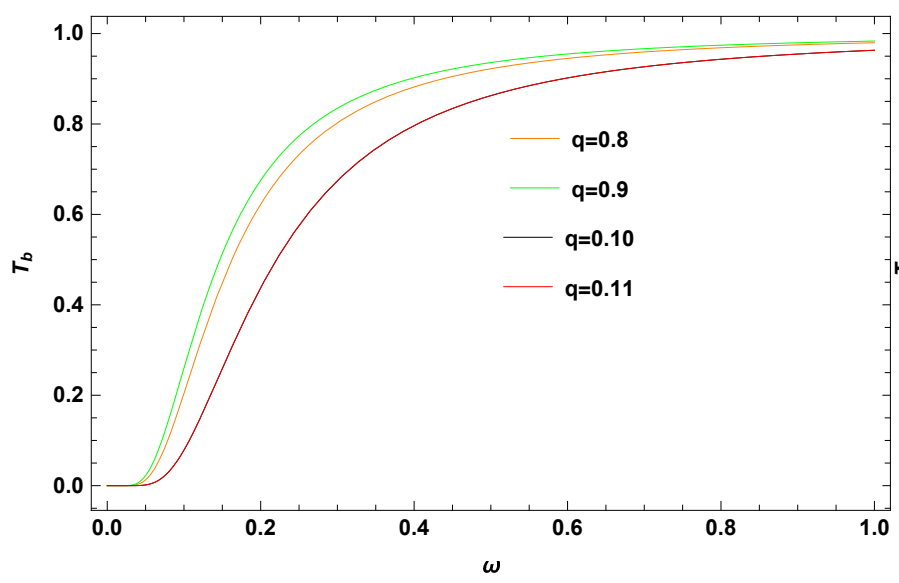

(i)

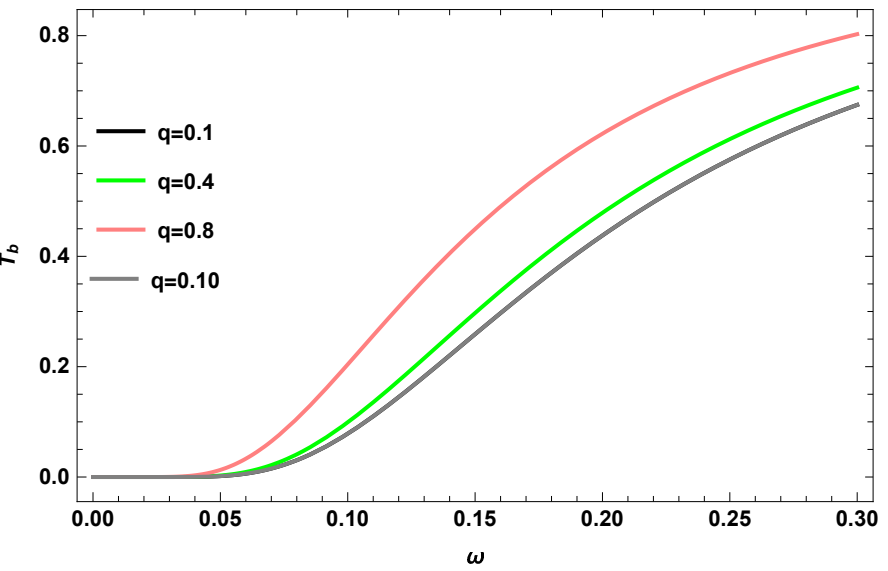

Figure 1: $T_{b}$ with respect to $\omega$.

- Fig 1 shows the behavior of $T_{b}$ in connection with $\omega$ when mass $m$, and $l$ are fixed and gives varation to $q$.

1. In the left figure, it can be seen that the bound $T_{b}$ is decreasing for high values of $q$. The domain is taken $0 \leq \omega \leq 1$.

2. In the right figure, it can be observed that obtained bound in case of different values of $q$ is decreasing. The domain is $0 \leq \omega \leq 0.30$.

Hence we concluded that in both cases the greybody bound $T_{b}$ is decrease.

\section{CONCLUSION}

The current paper, is concerning about exploration of deflection angle by MRBH for plasma and non-plasma mediums. For this purpose we use GBT and find the deflection angle of photon. Hence, our deflection angle is stated as:

$$
\begin{aligned}
\gamma & \approx \frac{(4 G m)}{b}+\frac{\left(3 G^{2} m^{2} \pi\right)}{4 b^{2}}-\frac{\left(3 G q^{2} \pi\right)}{4 b^{2}}-\frac{\left(8 G^{2} m q^{2}\right)}{3 b^{3}}-\frac{\left(75 G^{2} m^{2} \pi\right)}{8 b^{4}} \\
& -\frac{\left(45 G^{3} m^{2} q^{2} \pi\right)}{32 b^{4}}+\frac{\left(15 G^{2} q^{4} \pi\right)}{64 b^{4}}+\frac{\left(32 G^{2} m q^{2}\right)}{b^{5}}+\frac{\left(12 G^{3} m q^{4}\right)}{5 b^{5}} \\
& +\frac{\left(125 m^{2} G^{3} q^{2} \pi\right)}{8 b^{6}}+\frac{\left(525 G^{4} m^{2} q^{4} \pi\right)}{256 b^{6}}-\frac{\left(4192 G^{3} m q^{4}\right)}{245 b^{7}}-\frac{\left(43155 G^{4} m^{2} q^{4} \pi\right)}{2048 b^{8}} \\
& +\frac{\left(6111 G^{4} m^{2} q^{4} \pi\right)}{256 b^{10}}+\frac{\left(567 G^{4} l^{2} m^{2} q^{4} \pi\right)}{128 b^{10}} .
\end{aligned}
$$

The above angle of deflection is merged in to Schwarzschild-like deflection angle in to first order terms when $G=1, l=0$ and $q=0$. Also we study the graphical effect of different parameters on deflection angle by MRBH. The angle of deflection for 
plasma medium is defined as:

$$
\begin{aligned}
\gamma & \approx \frac{(4 G m)}{b}+\frac{\left(3 G^{2} m^{2} \pi\right)}{4 b^{2}}-\frac{\left(3 G q^{2} \pi\right)}{4 b^{2}}-\frac{\left(8 G^{2} m q^{2}\right)}{3 b^{3}}-\frac{\left(75 G^{2} m^{2} \pi\right)}{8 b^{4}}-\frac{\left(45 G^{3} m^{2} q^{2} \pi\right)}{32 b^{4}} \\
& +\frac{\left(15 G^{2} q^{4} \pi\right)}{64 b^{4}}+\frac{\left(32 G^{2} m q^{2}\right)}{b^{5}}+\frac{\left(12 G^{3} m q^{4}\right)}{5 b^{5}}+\frac{\left(125 m^{2} G^{3} q^{2} \pi\right)}{8 b^{6}}+\frac{\left(525 G^{4} m^{2} q^{4} \pi\right)}{256 b^{6}} \\
& -\frac{\left(4192 G^{3} m q^{4}\right)}{245 b^{7}}-\frac{\left(43155 G^{4} m^{2} q^{4} \pi\right)}{2048 b^{8}}+\frac{\left(6111 G^{4} m^{2} q^{4} \pi\right)}{256 b^{10}}+\frac{\left(567 G^{4} l^{2} m^{2} q^{4} \pi\right)}{128 b^{10}} \\
& +\frac{(2 G m) \omega^{2}}{b}-\frac{\left(15 G^{2} m^{2} \pi\right) \omega^{2}}{2 b^{4}}-\frac{\left(G^{2} m^{2} \pi\right) \omega^{2}}{2 b^{2}}+\frac{\left(80 G^{2} m q^{2}\right) \omega^{2}}{3 b^{5}}+\frac{\left(2 G^{2} m q^{2}\right) \omega^{2}}{b^{3}} \\
& -\frac{\left(G q^{2} \pi\right) \omega^{2}}{2 b^{2}}-\frac{\left(465 G^{3} m^{2} q^{2} \pi\right) \omega^{2}}{32 b^{6}}+\frac{\left(3 G^{3} m^{2} q^{2} \pi\right) \omega^{2}}{8 b^{4}}+\frac{\left(4112 G^{3} m q^{4}\right) \omega^{2}}{245 b^{7}}-\frac{\left(2 G^{3} m q^{4}\right) \omega^{2}}{3 b^{5}} \\
& -\frac{\left(3 G^{2} q^{4} \pi\right) \omega^{2}}{16 b^{4}}-\frac{\left(15435 G^{4} m^{2} q^{4} \pi\right) \omega^{2}}{256 b^{10}}+\frac{\left(14105 G^{4} m^{2} q^{4} \pi\right) \omega^{2}}{2048 b^{8}}-\frac{\left(45 G^{4} m^{2} q^{4} \pi\right) \omega^{2}}{128 b^{6}} \\
& +\frac{\left(945 G^{4} l^{2} m^{2} q^{4} \pi\right) \omega^{2}}{256 b^{10}} .
\end{aligned}
$$

When we consider $(\omega \rightarrow 0)$, then plasma is converted in to non-plasma.

The graphical effect of the deflection angle has also studied by changing the values of the charge and the impact parameter of the $\mathrm{BH}$. Then we calculated the equation for the rigorous bound $T_{b}$ that shows that the bound on the mass, gravitational constant, charge, $\omega$.

$$
T \geq \operatorname{sech}\left[\frac{0.625 l(l+1)+0.390625 G m-0.305176 G^{2} l^{2} m^{2}-0.16276 G q^{2}+0.198682 G^{2} l^{2} m q^{2}}{2 \omega}\right]^{2}
$$

The graphical behaviour of the bound shows that $T_{b}$ is decrease when we gives variation to the charge $q$.

[1] A. Einstein, Science 84, 506-507 (1936).

[2] A.Ricon, G.Panotopoulous, Phys.Dark Univ,100639 (2020).

[3] C. A. R. Herdeiro and J. P. S. Lemos, [arXiv:1811.06587 [physics.hist-ph]].

[4] B. P. Abbott et al. [LIGO Scientific and Virgo], Phys. Rev. Lett. 116, no.13, 131103 (2016).

[5] S. S. Li, S. Mao, Y. Zhao and Y. Lu, Mon. Not. Roy. Astron. Soc. 476, no.2, 2220-2229 (2018)

[6] M. Bartelmann and P. Schneider, Phys. Rept. 340, 291-472 (2001).

[7] P. V. P. Cunha and C. A. R. Herdeiro, Gen. Rel. Grav. 50, no.4, 42 (2018).

[8] J. Soldner, Ueber die Ablenkung eines Lichtstrals von seiner geradlinigen Bewegung, durch die Attraktion eines Weltkörpers, an welchem er nahe vorbei geht. Berliner Astronomisches Jahrbuch,161-172.cc (1804).

[9] F. W. Dyson, A. S. Eddington and C. Davidson, Phil. Trans. Roy. Soc. Lond. A 220, 291-333 (1920).

[10] E. F. Eiroa, G. E. Romero and D. F. Torres, Phys. Rev. D 66, 024010 (2002).

[11] C. R. Keeton, C. S. Kochanek and E. E. Falco, Astrophys. J. 509, 561-578 (1998).

[12] M. Sharif and S. Iftikhar, Astrophys. Space Sci. 361, no.1, 36 (2016).

[13] K. S. Virbhadra, D. Narasimha and S. M. Chitre, Astron. Astrophys. 337, 1-8 (1998).

[14] R. Shaikh, P. Kocherlakota, R. Narayan and P. S. Joshi, Mon. Not. Roy. Astron. Soc. 482, no.1, 52-64 (2019).

[15] R. Shaikh, P. Kocherlakota, R. Narayan and P. S. Joshi, Mon. Not. Roy. Astron. Soc. 482, no.1, 52-64 (2019).

[16] S. U. Islam, R. Kumar and S. G. Ghosh, JCAP 09, 030 (2020)

[17] R. Kumar, S. G. Ghosh and A. Wang, Phys. Rev. D 101, no.10, 104001 (2020)

[18] T. Manna, F. Rahaman, S. Molla, J. Bhadra and H. H. Shah, Gen. Rel. Grav. 50, no.5, 54 (2018).

[19] P. K. F. Kuhfittig, Eur. Phys. J. C 74, no.99, 2818 (2014).

[20] F. Rahaman, M. Kalam and S. Chakraborty, Chin. J. Phys. 45, 518 (2007).

[21] G. W. Gibbons and M. C. Werner, Class. Quant. Grav. 25, 235009 (2008).

[22] M. C. Werner, Gen. Rel. Grav. 44, 3047-3057 (2012).

[23] A. Ishihara, Y. Suzuki, T. Ono, T. Kitamura and H. Asada, Phys. Rev. D 94, no.8, 084015 (2016)

[24] G. Crisnejo and E. Gallo, Phys. Rev. D 97, no.12, 124016 (2018).

[25] A. Ishihara, Y. Suzuki, T. Ono and H. Asada, Phys. Rev. D 95, no.4, 044017 (2017).

[26] A. Övgün, Phys. Rev. D 98, no.4, 044033 (2018).

[27] A. Övgün, Phys. Rev. D 99, no.10, 104075 (2019).

[28] A. Övgün, Universe 5, no.5, 115 (2019).

[29] K. Jusufi, M. C. Werner, A. Banerjee and A. Övgün, Phys. Rev. D 95, no.10, 104012 (2017).

[30] T. Ono, A. Ishihara and H. Asada, Phys. Rev. D 99, no.12, 124030 (2019). 
[31] K. de Leon and I. Vega, Phys. Rev. D 99, no.12, 124007 (2019).

[32] K. Jusufi and A. Övgün, Phys. Rev. D 97, no.2, 024042 (2018).

[33] T. Ono, A. Ishihara and H. Asada, Phys. Rev. D 96, no.10, 104037 (2017)

[34] K. Jusufi, A. Ovgün and A. Banerjee, Phys. Rev. D 96, no.8, 084036 (2017).

[35] A. Övgün, G. Gyulchev and K. Jusufi, Annals Phys. 406, 152-172 (2019).

[36] H. Arakida, Gen. Rel. Grav. 50, no.5, 48 (2018).

[37] K. Jusufi and A. Ovgün, Phys. Rev. D 97, no.6, 064030 (2018).

[38] T. Ono, A. Ishihara and H. Asada, Phys. Rev. D 98, no.4, 044047 (2018).

[39] K. Jusufi, A. Övgün, J. Saavedra, Y. Vásquez and P. A. González, Phys. Rev. D 97, no.12, 124024 (2018).

[40] T. Ono, A. Ishihara and H. Asada, Phys. Rev. D 99, no.12, 124030 (2019).

[41] W. Javed, R. Babar and A. Övgün, Phys. Rev. D 99, no.8, 084012 (2019).

[42] K. Jafarzade, M. Kord Zangeneh and F. S. N. Lobo, JCAP 04, 008 (2021).

[43] K. Takizawa, T. Ono and H. Asada, Phys. Rev. D 101, no.10, 104032 (2020).

[44] Y. Kumaran and A. Övgün, Chin. Phys. C 44, no.2, 025101 (2020).

[45] Z. Li and A. Övgün, Phys. Rev. D 101, no.2, 024040 (2020).

[46] Z. Li, G. Zhang and A. Övgün, Phys. Rev. D 101, no.12, 124058 (2020).

[47] A. Övgün, Turk. J. Phys. 44, no.5, 465-471 (2020).

[48] Y. Kumaran and A. Övgün, Turk. J. Phys. 45, 247-267 (2021).

[49] M. Okyay and A. Övgün, JCAP 01, no.01, 009 (2022).

[50] R. C. Pantig, P. K. Yu, E. T. Rodulfo and A. Övgün, Annals of Physics 436, 168722 (2022).

[51] K. Takizawa, T. Ono and H. Asada, Phys. Rev. D 102, no.6, 064060 (2020).

[52] T. Ono and H. Asada, Universe 5, no.11, 218 (2019).

[53] Q. M. Fu, L. Zhao and Y. X. Liu, Phys. Rev. D 104, no.2, 024033 (2021).

[54] A. Ishihara, Y. Suzuki, T. Ono and H. Asada, Phys. Rev. D 95, no.4, 044017 (2017).

[55] H. El Moumni, K. Masmar and A. Övgün, [arXiv:2008.06711 [gr-qc]].

[56] W. Javed, R. Babar and A. Övgün, Phys. Rev. D 100, no.10, 104032 (2019).

[57] W. Javed, J. Abbas and A. Övgün, Eur. Phys. J. C 79, no.8, 694 (2019).

[58] W. Javed, j. Abbas and A. Övgün, Phys. Rev. D 100, no.4, 044052 (2019).

[59] W. Javed, A. Hamza and A. Övgün, Phys. Rev. D 101, no.10, 103521 (2020).

[60] W. Javed, M. B. Khadim, A. Övgün and J. Abbas, Eur. Phys. J. Plus 135, no.3, 314 (2020).

[61] W. Javed, M. B. Khadim and A. Övgün, Eur. Phys. J. Plus 135, no.7, 595 (2020).

[62] W. Javed, A. Hamza and A. Övgün, Mod. Phys. Lett. A 35, no.39, 2050322 (2020).

[63] W. Javed, A. Hamza and A. Övgün, Universe 7, no.10, 385 (2021).

[64] W. Javed, J. Abbas, Y. Kumaran and A. Övgün, Int. J. Geom. Meth. Mod. Phys. 18, no.01, 2150003 (2021).

[65] W. Javed, J. Abbas and A. Övgün, Annals Phys. 418, 168183 (2020).

[66] S. Hensh, A. Abdujabbarov, J. Schee and Z. Stuchlík, Eur. Phys. J. C 79, no.6, 533 (2019).

[67] S. W. Hawking, Commun. Math. Phys. 43, 199-220 (1975) [erratum: Commun. Math. Phys. 46, 206 (1976)].

[68] S. Fernando, Gen. Rel. Grav. 37, 461-481 (2005).

[69] G. Panotopoulos and A. Rincón, Phys. Rev. D 97, no.8, 085014 (2018).

[70] J. M. Maldacena and A. Strominger, Phys. Rev. D 55, 861-870 (1997).

[71] D. Ida, K. y. Oda and S. C. Park, Phys. Rev. D 67, 064025 (2003) [erratum: Phys. Rev. D 69, 049901 (2004)].

[72] M. Cvetic and F. Larsen, Phys. Rev. D 56, 4994-5007 (1997).

[73] T. Harmark, J. Natario and R. Schiappa, Adv. Theor. Math. Phys. 14, no.3, 727-794 (2010).

[74] S. S. Gubser and I. R. Klebanov, Phys. Rev. Lett. 77, 4491-4494 (1996).

[75] I. R. Klebanov and S. D. Mathur, Nucl. Phys. B 500, 115-132 (1997).

[76] H. W. Lee and Y. S. Myung, Phys. Rev. D 58, 104013 (1998).

[77] S. Chen and J. Jing, Phys. Lett. B 691, 254-260 (2010).

[78] R. A. Konoplya and A. F. Zinhailo, Phys. Lett. B 810, 135793 (2020).

[79] P. Boonserm and M. Visser, Annals Phys. 323, 2779-2798 (2008).

[80] P. Boonserm, [arXiv:0907.0045 [math-ph]].

[81] P. Boonserm and M. Visser, Phys. Rev. D 78, 101502 (2008).

[82] T. Ngampitipan and P. Boonserm, Int. J. Mod. Phys. D 22, 1350058 (2013).

[83] P. Boonserm, T. Ngampitipan and P. Wongjun, Eur. Phys. J. C 78, no.6, 492 (2018).

[84] P. Boonserm, T. Ngampitipan and M. Visser, JHEP 03, 113 (2014).

[85] P. Boonserm, A. Chatrabhuti, T. Ngampitipan and M. Visser, J. Math. Phys. 55, 112502 (2014).

[86] T. Ngampitipan and P. Boonserm, J. Phys. Conf. Ser. 435, 012027 (2013).

[87] P. Boonserm, T. Ngampitipan and P. Wongjun, Eur. Phys. J. C 79, no.4, 330 (2019).

[88] S. I. Kruglov, Int. J. Mod. Phys. A 36, no.21, 2150158 (2021). 